\title{
Human responses against HER-2-positive cancer cells in human immune system-engrafted mice
}

\author{
C De Giovanni ${ }^{1,2}$, G Nicoletti ${ }^{3}$, L Landuzzi ${ }^{3}$, F Romani ${ }^{4}$, S Croci $^{1}$, A Palladini ${ }^{1}$, A Murgo', A Antognoli', \\ ML lanzano', V Stivani', V Grosso', M lezzi ${ }^{5}$, L Stramucci ${ }^{5}$, E Barbieri ${ }^{2,6}$, RM Lemoli ${ }^{2,7}$, P Nanni $^{*, 1,2}$ and \\ P-L Lollini ${ }^{2,7}$
}

'Department of Experimental Pathology, Cancer Research Section, Alma Mater Studiorum Università di Bologna, Viale Filopanti 22, I-40 I 26 Bologna, Italy; ' Centro Interdipartimentale di Ricerche sul Cancro 'Giorgio Prodi', Alma Mater Studiorum Università di Bologna, Via Massarenti 9, I-40 I 38 Bologna, Italy; ${ }^{3}$ Laboratory of Experimental Oncology, Rizzoli Orthopedic Institute, Via di Barbiano I/I0, 1-40/36 Bologna, Italy; ${ }^{4}$ Direzione Fisica Sanitaria, Azienda Ospedaliero-Universitaria di Bologna, Policlinico S. Orsola Malpighi, Via Massarenti 9, I-40138 Bologna, Italy; ${ }^{5}$ CESI Aging Research Center, G. D’Annunzio University, Via Colle dell'Ara, I-660 I 3 Chieti Scalo, Chieti, Italy; ${ }^{6}$ Dipartimento Clinico di Scienze Radiologiche e Istocitopatologiche, Alma Mater Studiorum Università di Bologna, Via Massarenti 9, I-40 I 38 Bologna, Italy; 'Department of Hematology and Oncologic Sciences 'L. e A. Seragnoli', Alma Mater Studiorum Università di Bologna, Via Massarenti 9, I-40I 38 Bologna, Italy

BACKGROUND: Human immune system (HIS)-engrafted mice are new tools to investigate human immune responses. Here, we used HIS mice to study human immune responses against human HER-2-positive cancer cells and their ability to control tumour growth and metastasis.

METHODS: BALB/c Rag2 ${ }^{-1-}, 112 \mathrm{rg}^{-1-}$ mice were engrafted with $\mathrm{CD} 34^{+}$or $\mathrm{CDI} 33^{+}$human cord blood hematopoietic stem cells (HSC) and vaccinated with human HER-2-positive cancer cells SK-OV-3 combined to human IL-I2.

RESULTS: Both $\mathrm{CD}_{3} 4^{+}$or $\mathrm{CDI} 33^{+}$human HSC gave long-term engraftment and differentiation, both in peripheral blood and in lymphoid organs, and production of human antibodies. Vaccinated mice produced specific anti-HER-2 human IgG. An s.c. SK-OV-3 challenge was significantly inhibited (but not abolished) in both vaccinated and non-vaccinated HIS mice. Tumours were heavily infiltrated with human and murine cells, mice showed NK cells and production of human interferon- $\gamma$, that could contribute to tumour growth inhibition. Vaccinated HIS mice showed significantly inhibited lung metastases when compared with non-vaccinated HIS mice and to non-HIS mice, along with higher levels of tumour-infiltrating human dendritic cells.

CONCLUSION: Anti-HER-2 responses were elicited through an adjuvanted allogeneic cancer cell vaccine in HIS mice. Human immune responses elicited in HIS mice effectively inhibited lung metastases.

British Journal of Cancer (2012) I 07, 1302-1309. doi:I0.1038/bjc.2012.394 www.bjcancer.com

Published online 28 August 2012

(C) 2012 Cancer Research UK

Keywords: HER-2; IL-I2; cancer vaccine; human immune system mice; hematopoietic stem cells; metastasis

Attempts to obtain mice reconstituted with a human immune system (HIS) mice, also referred to as HIS mice, date back to the end of 1980 s, when mice carrying the Prkdc scid mutation were first used as recipients of human progenitors, with limited success (Shultz et al, 2007). In the last decade, mice with engineered combined immunodeficiencies were obtained and a more efficient engraftment of human hematopoietic stem cells (HSC) was described (Shultz et al, 2007; Manz and Di Santo, 2009; Brehm et al, 2010). Even though the full human reconstitution is generally not achieved, HIS mice have already given valuable results for a better understanding of the differentiation potential of human immune populations (Huntington and Di Santo, 2008; Meek et al, 2010) and for the study of viral human pathogens (Legrand et al, 2009). Moreover, HIS mice are interesting models for human vaccine development (Koo et al, 2009; Becker et al, 2010). Human immune system mice, could provide new experimental models to

*Correspondence: Dr P Nanni; E-mail: patrizia.nanni@unibo.it Received 9 May 2012; revised 26 July 2012; accepted 6 August 2012; published online 28 August 2012 study the relationships between human tumours and human immune effectors (Brehm et al, 2010; Wege et al, 2011).

$\mathrm{BALB} / \mathrm{c} \mathrm{Rag2}{ }^{-/-} ; \mathrm{Il}_{2 \mathrm{rg}^{-/-}}$(hereafter referred to as BRG mice), display a severe combined immunodeficiency, with absence of T, B and NK cells (Nomura et al, 2008). These mice can unveal growth and metastatic ability of human xenografts, otherwise not apparent in mice with partial immunodeficiencies (Nanni et al, 2010). BALB/c Rag2 ${ }^{-I_{-}} ; \mathrm{Il}_{2} \mathrm{rg}^{-/-}$mice have been effectively used to obtain HIS mice (Traggiai et al, 2004; Brehm et al, 2010) and to test antitumour activity of human immune effectors (Thiel et al, 2011). We exploited these features to study the possibility to induce immune responses against human cancer cells by human lymphocytes and their ability to control tumour growth and metastasis.

As the tumoural counterpart, we selected a HER-2-positive human cancer cell line. Therapeutic targeting of HER-2-overexpressing cancers with the humanised monoclonal antibody trastuzumab has already entered the clinical practice, but the search for active immunisation strategies against HER-2 is also pursued (Wei et al, 2008). In transgenic models of HER-2-driven mammary carcinogenesis, immune targeting of HER-2 with both 
active and passive approaches has shown preventive and therapeutic activity (Lollini et al, 2011). Transgenic models are suitable to study, at best, the murine immune response against the transgenic human HER-2 molecule, but cellular or soluble immune effectors elicited in transgenic mice are not of human origin.

In the first part of this study we investigated the reconstituting ability of two types of HSC sorted from human cord blood: CD34 and $\mathrm{CD} 133^{+}$cells. CD $34^{+}$progenitors have been extensively used to obtain HIS mice, see for BRG the pioneering study of Traggiai et al (2004). CD133 ${ }^{+}$HSC engraftment was reported after in vitro culture in growth factor-supplemented medium (Drake et al, 2011), whereas studies with freshly isolated CD133 + HSC in BRG mice and a direct comparison with CD34 + HSC engraftment ability have not been reported. Subsequently, we investigated the ability to elicit an immune response against HER-2-positive human cancer cells.

\section{MATERIALS AND METHODS}

\section{Generation of HIS mice}

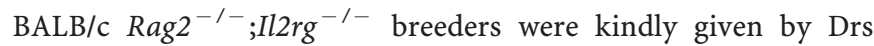
$\mathrm{T}$ Nomura and $\mathrm{M}$ Ito of the Central Institute for Experimental Animals (Kawasaki, Japan), then mice were bred in our animal facilities under sterile conditions. Human immune system mice were generated as described (Traggiai et al, 2004). For details see Supplementary Materials and Methods. Cord blood mononuclear cells were enriched by Ficoll-Hypaque (Amersham Bioscience, Piscataway, NJ, USA) and then processed by MiniMacs highgradient magnetic separation column (Miltenyi Biotec, Bergisch Gladbach, Germany) to obtain highly purified cells (mean purity $93 \% \pm 6 \%$ ) as previously described (Rossi et al, 2007). For challenge experiments, $\mathrm{CD} 34^{+}$or $\mathrm{CD} 133^{+}$cells were purchased from AllCells (Emeryville, CA, USA). All the experiments were authorised by the Animal Care and Use Committee of the University of Bologna, and done according to Italian and European guidelines.

\section{Monitoring of HIS mice engraftment}

To assess the level of engraftment and human leukocyte populations, HIS mice were bled every 5 weeks from a tail vein. At the time of killing, the bone marrow (from a femur), spleen, thymus and mesenteric lymph node were collected, spleen cell suspensions were subjected to red blood cell lysis. Cell yield of each organ was determined by staining with Turk's solution and counting in a hemocytometer. Human leukocyte populations were analysed by direct immunofluorescence using PE- or FITCconjugated antibodies purchased from BD Pharmingen, San Diego, CA, USA). PE- and FITC-conjugated mouse IgG1 isotype control antibodies were also used. Fluorescence-activated cell sorting (FACS) analysis was performed with FACScan (Becton Dickinson, St Jose, CA, USA).

\section{Vaccination and tumour challenge}

At 6 weeks HIS mice were distributed into two groups with matched levels of CD45 + human circulating cells. A group was subjected to two cycles of anti-HER-2 cell vaccination according to a schedule previously reported highly effective in HER-2/neu transgenic models (Nanni et al, 2001). Cell vaccine consisted of the human HER-2-positive SK-OV-3 cancer cells, with a high membrane expression of human HER-2. SK-OV-3 cell line was authenticated by DNA fingerprinting on the 11 November 2010 (performed by DSMZ, Braunschweig, Germany). Mice received four i.p. vaccinations distributed in 2 weeks consisting of $2 \times 10^{6}$ mitomycin-treated cells (to block proliferation) in $0.4 \mathrm{ml}$ PBS. In the third week mice received five times daily administration of recombinant human IL-12 (PeproTech, Rocky Hill, NJ, USA) in 0.2 $\mathrm{ml}$ PBS supplemented with $0.01 \%$ mouse serum albumin (Sigma Aldrich, St Louis, MO, USA). The dose of IL-12 was 50 ng per mouse per day in the first 4-week cycle and $100 \mathrm{ng}$ per mouse per day in all subsequent cycles. The fourth-week mice received no treatment. The 4 -week vaccination cycle was repeated twice. Nonvaccinated group received the injection of PBS or PBS supplemented with mouse serum albumin.

Vaccinated and non-vaccinated HIS mice, at the age of 17 weeks, received the s.c. injection of $10^{5}$ live SK-OV-3 cells in $0.2 \mathrm{ml}$ PBS in a flank. Mice were monitored twice weekly for tumour growth by measuring tumour volume, calculated as $\pi \cdot[\sqrt{ }(a \cdot b)]^{3} / 6$, where $a=$ maximal tumour diameter and $b=$ tumour diameter perpendicular to $a$. Mice were killed 40 days after tumour challenge, subjected to an accurate necropsy, tumours and lymphoid organs were collected and processed as reported in other sections. Lungs were fixed in Fekete's solution and metastases were counted using a dissection microscope.

\section{Tumour-infiltrating populations}

For histological and immunohistochemical analyses tumours were divided in two pieces: half was fixed in buffered formalin and embedded in paraffin, half was fixed in periodate lysine paraformaldehyde, cryoprotected in sucrose and frozen in OCT. For details see Supplementary Materials and Methods.

\section{Human cytokine production}

Cells harvested from lymphoid organs were cultured in vitro for 6 days in RPMI 1640 supplemented with 10\% fetal bovine serum and with recombinant human IL-2 (50 units $\mathrm{ml}^{-1}$, PeproTech). Concentration of human cytokines in culture supernatants as well as in plasma samples was determined with the Bio-Plex 200 suspension array system (Bio-Rad, Milan, Italy) (De Giovanni et al, 2009). Production of human interferon (IFN)- $\gamma$ was also determined by ELISA (Quantikine Human IFN- $\gamma$, R\&D Systems, Minneapolis, MN, USA).

\section{Human antibodies}

Concentration of total human IgM and IgG in plasma from HIS mice was determined by ELISA (Human IgM and Human IgG ELISA quantitation kits, Bethyl Laboratories, Montgomery, TX, USA). Human reference serum $\left(0.38 \mathrm{mg} \mathrm{ml}^{-1} \mathrm{IgM}\right.$ and $4 \mathrm{mg} \mathrm{ml}^{-1}$ IgG, Bethyl Laboratories) was used as a standard. Induction of human antibodies recognising human HER-2/neu was investigated by immunoprecipitation of HER-2-positive cell lysates with plasma samples followed by western blot analysis. For details on detection and in vitro activities see Supplementary Materials and Methods.

\section{Statistical analysis}

Engraftment data, antibody levels, cytokine production, tumour volumes and metastases were compared by the Student's $t$-test and by the non-parametric Wilcoxon rank sum test. Frequencies were compared by the $\chi^{2}$ Fisher's exact test.

\section{RESULTS}

\section{Engraftment of human $\mathrm{CD}_{3}{ }^{+}$and $\mathrm{CD} 33^{+} \mathrm{HSC}$}

Within $24-48 \mathrm{~h}$ after birth, BRG mice were subjected to sublethal irradiation and to the intrahepatic injection of $1-2 \times 10^{5} \mathrm{CD}_{3} 4^{+}$or $\mathrm{CD} 33^{+}$human cord blood progenitors. At 5 weeks of age most mice (about $90 \%$ ) showed at least $10 \%$ of circulating human $\mathrm{CD} 5^{+}$cells (Supplementary Figure 1A). The level of engraftment did not correlate with the number of HSC injected (Supplementary 
Figure 1B). These mice will be hereafter referred to as $\mathrm{rCD} 34$ or rCD133, according to the HSC population used for reconstitution, or HIS mice as a whole. The two groups of HIS mice showed similar mean values of circulating human CD45 + and ranges, reaching maximal values of $78 \%$ for $\mathrm{rCD} 34$ and $71 \%$ for rCD133 (Supplementary Figure 1A). A long-term persistence of engraftment was obtained, with total circulating human CD45+ over $20-25 \%$ up to 20 weeks of age (Figure 1 and Supplementary Figure 2), and a slightly higher persistence for rCD133. Along time, the rise of circulating human CD3 + cells (up to about $30-40 \%$ of human CD45 + cells at 20 week) and a parallel decrease of human $\mathrm{CD} 19+$ cells were found (Figure 1 and Supplementary Figure 2). A significantly higher level of $\mathrm{CD} 19+$ cells was observed in rCD133 mice at 10 week.

Human IgM and IgG immunoglobulins became detectable in mice serum 10 weeks after transplantation (Figure 1); rCD133 mice showed significantly higher serum IgM levels at some time points, but lower IgG levels. The level of early engraftment (at 5-10 week) in rCD34 mice strongly correlated with the rise of human IgM levels, whereas less significant correlations were observed in rCD133 mice and in both HIS mice for IgG levels (data not shown).

Lymphoid organs of rCD34 and rCD133 mice showed similar levels of engrafted human CD $45+$ cells, up to $50 \%$ for the spleen and bone marrow and about $85-95 \%$ for thymus and mesentheric lymph node (Supplementary Figure 3). Engrafting of human cells also caused an increase in the total number of cells recovered from the thymus, mesentheric lymph node and spleen of HIS mice, whereas non-HIS BRG mice have atrophic or even undetectable thymus and mesentheric lymph node and a low spleen cellularity (Supplementary Figure 3).

In conclusion, our data demonstrate, for the first time, the long-term engraftment capacity of $\mathrm{CD}_{13} 3^{+} \mathrm{HSC}$ in BRG mice.

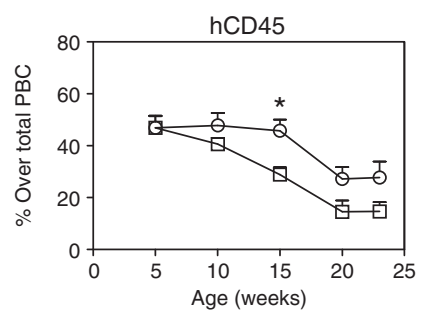

Ð rCD34 mice
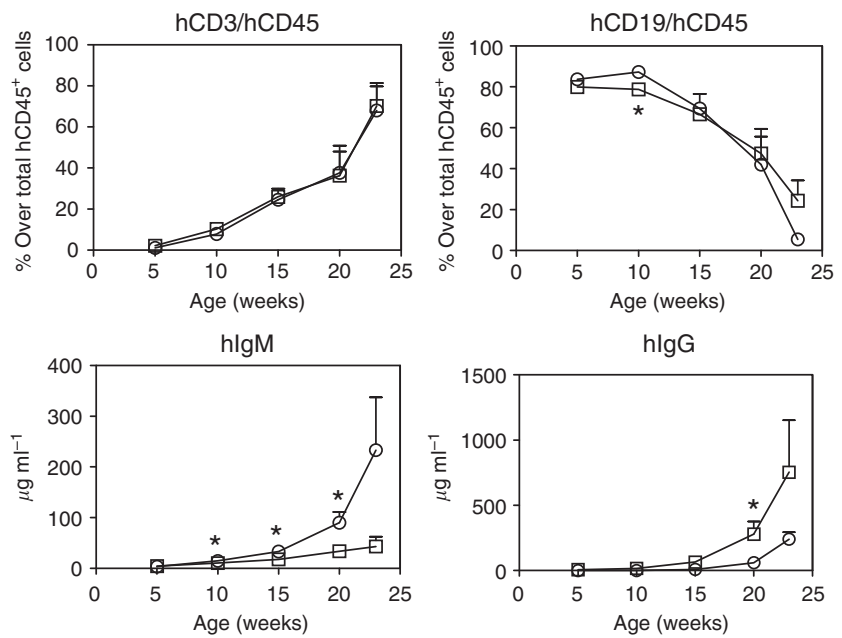

Figure I Kinetics of human populations in peripheral blood and of lgM and IgG human antibody levels in mice engrafted with human CD34 + or CDI33 + HSC (indicated as rCD34 or rCDI33, respectively). Mean and s.e. is shown (7-28 mice per group). ${ }^{*} P<0.05$ at least at Wilcoxon non-parametric rank sum test between rCD34 and rCDI33.
When compared with $\mathrm{CD} 34^{+} \mathrm{HSC}, \mathrm{CD} 133^{+}$cells demonstrated a slightly higher total and B lymphoid reconstitution.

\section{Induction of a human humoral response against HER-2-positive cancer cells}

To study whether HIS mice can develop an immune response against human cancer cells, at 6 weeks of age HIS rCD34 or rCD133 mice were distributed into two arms with matched levels of CD45 + human circulating cells (Figure 2A). A group did not receive any further treatment (non-vaccinated group) whereas the other group was vaccinated with proliferation-blocked human HER-2-positive SK-OV-3 cancer cells combined to recombinant human IL-12.

Throughout the vaccination courses, the levels of circulating human CD45 + cells as well as those of other human immune subpopulations (Figure 2B) showed kinetics similar to those of non-vaccinated HIS mice. A similar rise in total human IgG and IgM antibodies was found at 15 weeks of age in non-vaccinated and vaccinated $\mathrm{rCD} 34$ mice (Figure $3 \mathrm{~A}$ ). Therefore, vaccination per se did not affect persistence of human cell engraftment, differentiative ability and production of total antibodies.

At 15 weeks of age sera from vaccinated rCD34 mice showed specific anti-HER-2 IgG antibodies, based on their ability to immunoprecipitate HER-2 from a SK-OV-3 cell lysate (Figure 3B, lane 4). Sera from non-vaccinated $\mathrm{rCD} 34$ mice before challenge did not show any anti-HER-2 immunoprecipitating activity (Figure 3B, lane 3). Therefore, vaccination was able to elicit production of specific anti-HER-2 human IgG antibodies.

\section{Growth and metastasis of HER-2-positive human cancer cells in HIS mice}

After completion of two vaccination cycles, HIS mice received the s.c. injection of live SK-OV-3 cancer cells. Tumour growth in HIS mice was compared with that of the two groups of nonreconstituted age-matched control mice, run in parallel: untreated BRG mice and mice who only received the same sublethal neonatal irradiation used before reconstitution. A significant inhibition of tumour growth was observed in vaccinated as well as in nonvaccinated $\mathrm{rCD} 34$ mice (Figure 4). A similar behaviour was found in $\mathrm{rCD} 133$ mice (data not shown). As SK-OV-3 cells release cytokines and growth factors, such as IL-6, IL- 8 and VEGF (our unpublished results and literature data) (Melani et al, 1995; Keyes et al, 2003; Wang et al, 2010), we studied their serum levels in tumour-bearing mice. Human VEGF sera levels in HIS mice were significantly lower than that in non-reconstituted mice (Supplementary Figure 4), in agreement with the decreased tumour volume. On the contrary, human IL-6 and IL-8 were not significantly different (Supplementary Figure 4), likely due to a balance between cytokines produced by tumours and those produced by the human cells of reconstituted mice. In conclusion, HIS mice showed an inhibited tumour growth, but vaccination did not give a significant contribution over reconstitution to control the s.c. tumour.

Almost all rCD34 remained alive up to 40 days after tumour challenge, and were then killed to analyse metastatic dissemination of human SK-OV-3 tumour cells and parameters of the immune response. SK-OV-3 cells gave rise to lung metastases in $83-100 \%$ non-vaccinated mice (either untreated BRG and $\mathrm{rCD} 34$ ), whereas a significant inhibition of lung metastases was found in vaccinated rCD34 mice (Table 1).

To analyse the immune response elicited in HER-2-positive tumour-bearing vaccinated and non-vaccinated HIS mice, at the time of their killing ( 23 weeks of age) we studied human populations in peripheral blood, in the tumour and in lymphoid organs, total and specific antibody production, and cytokine production by human cells. 
A

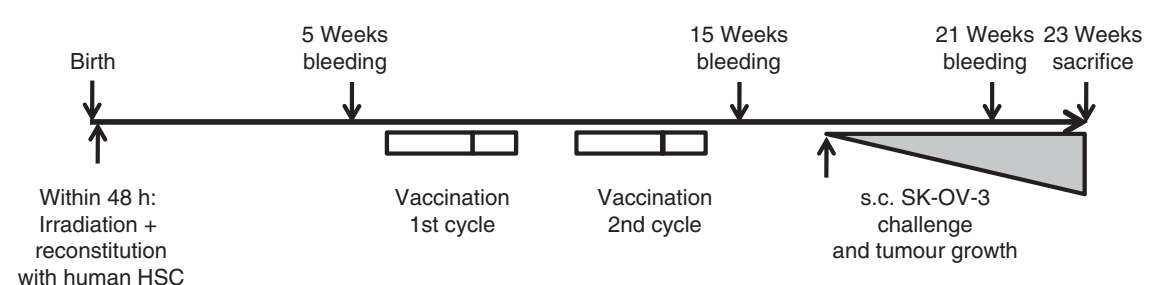

B
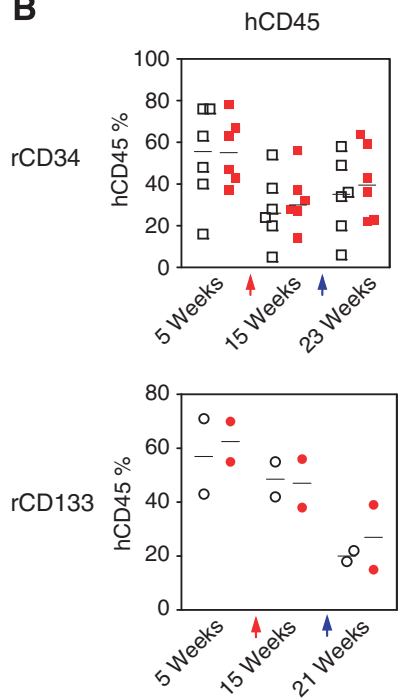
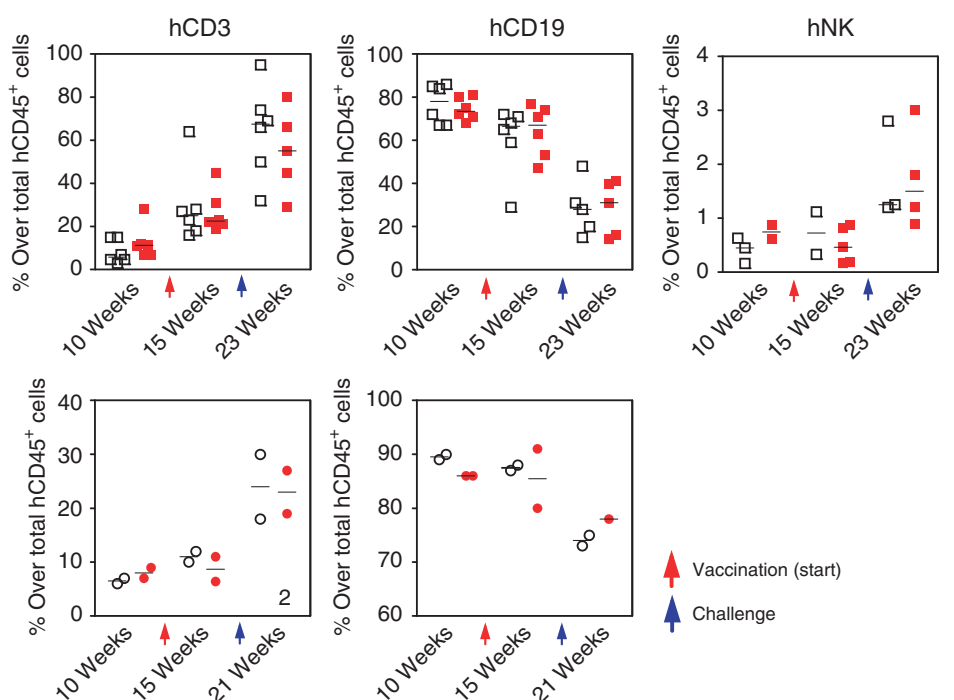

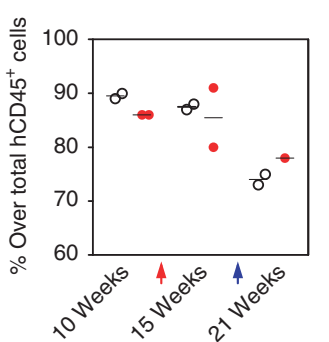

Figure 2 Vaccination and challenge with HER-2-positive human cancer cells in HIS mice. (A) Schedule of treatments. (B) Circulating human populations in HIS mice subjected to the vaccination-challenge experiment, tested at the following times: 5 week, assessment of human engrafting level and distribution of HIS mice in two matched groups; 15 weeks, after the completion of two vaccination cycles; 2I-23 weeks, after tumour growth (sacrifice time). Open symbols: non-vaccinated; closed red symbols: vaccinated. Values of individual mice are shown. Continuous horizontal lines indicate median values.

Vaccination-challenge procedure did neither modify the $\mathrm{CD} 45+$ level nor the frequency of human CD3 + and CD19+ populations in lymphoid organs of rCD34 mice nor the corresponding absolute cell yield, with the exception of a higher cell yield in thymus of vaccinated mice (Supplementary Table 1). The NK cells, almost undetectable before challenge, increased during challenge of both vaccinated and non-vaccinated HIS mice up to about $2 \%$ of total peripheral blood cells (Figure 2B), and reached $7-8 \%$ in mesentheric lymph node (data not shown). Human plasma cells (cells positive for both human CD38 and CD138) were found in the spleen of challenged mice at heterogeneous levels: individual total IgG serum level was correlated to splenic plasma cell frequency (Supplementary Figure 5).

All tumours showed a rich human $\mathrm{T}$ lymphocyte infiltrate (Figure 5), often with a perivascular arrangement similar to what is seen in allograft rejection, mainly composed by cytotoxic $\mathrm{T}$ cells and, at a lower frequency, by helper and regulatory $\mathrm{T}$ cells. Numerous NK cells were also consistently present. Human CD11cpositive dendritic cells were found at heterogeneous levels. A semiquantitative evaluation of tumour-infiltrating human populations showed an increased level of dendritic cells in rCD34 vaccinated over non-vaccinated mice, and such difference approached statistical significance (Table 2). CD45R + B cells were not found in tumours (data not shown). A very rich murine leukocyte infiltrate with phagocytic features composed of neutrophils, macrophages and dendritic cells was also present in all the tumours (Figure 5).

After challenge, total human IgG levels in vaccinated rCD34 mice reached significantly higher and less dispersed levels than in non-vaccinated rCD34 mice (Figure 3A). Challenge elicited high levels of specific anti-HER-2 IgG antibodies in vaccinated rCD34 mice (Figure 3B, lanes $8-10$ ), but provided the antigenic stimuli to induce trace amounts of anti-HER-2 IgG antibodies also in non-vaccinated mice (Figure 3B, lanes 5-7). Sera containing antiHER-2 antibodies showed growth-inhibiting and ADCC activities against HER-2-positive human cancer cells (Figure 3C). Comparing the data obtained pre- and post challenge, challenge boosted the production of anti-HER-2 human IgG antibodies elicited by vaccination, but could even induce it at a lower level in non-vaccinated HIS mice.

Release of human cytokines by the spleen cells, cultured alone or in the presence of proliferation-blocked vaccine cells, was studied as a parameter of cell-mediated immune response. Human IFN- $\gamma$ was spontaneously released at variable levels by the spleen cells of challenged rCD34 mice (Figure 6), whereas it had not been detected in rCD34 mice killed just after the completion of the vaccination (data not shown). The in vitro restimulation with vaccine cells induced only a slight, not significant increase of IFN- $\gamma$ release (Figure 6, vaccine-restimulated groups). Significant levels of the secondary IFN- $\gamma$-inducible cytokine IP-10 were also detected, whereas only trace amounts of TNF- $\alpha$ were found at similar levels in vaccinated and non-vaccinated $\mathrm{rCD} 34$ mice (Figure 6).

\section{DISCUSSION}

In this paper, we studied HIS mice engrafted with two different human HSC: $\mathrm{CD}_{3}{ }^{+}$and $\mathrm{CD} 133^{+}$cells. We then studied the ability of HIS mice to elicit a human immune reaction towards human HER-2-positive cancer cells.

Although the ability of $\mathrm{CD}^{+} 4^{+}$human cord blood HSC to reconstitute a human immune system in BRG mice has been extensively described (Brehm et al, 2010), freshly isolated CD133 ${ }^{+}$ cord blood progenitors were not studied. In humans, CD133 ${ }^{+}$ HSC have been already used to restore bone marrow function in 
A
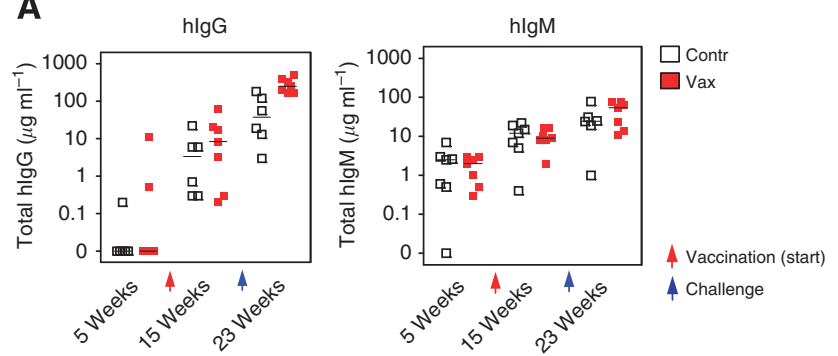

B

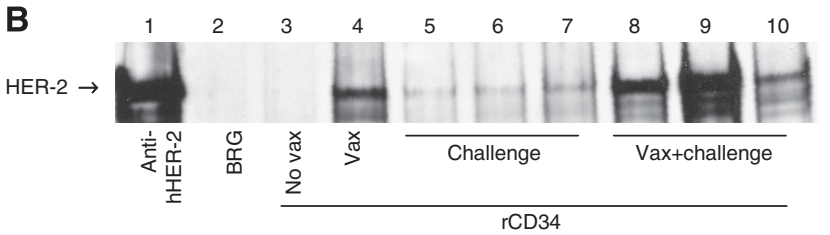

C
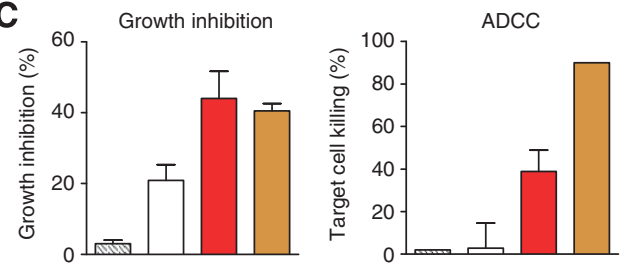

Sera:

BRG

rCD34, no vax rCD34, vax $\square$ Trastuzumab

Figure 3 Total and HER-2-specific human antibodies in rCD34 mice. (A). Total IgG (left) and total IgM (right) serum levels. Open symbols: non-vaccinated; closed red symbols: vaccinated. Values of individual mice are shown. Continuous horizontal lines indicate median values. (B) AntiHER-2 antibodies detected through immunoprecipitation. Sera (a volume containing $1.5 \mu \mathrm{g}$ total human $\operatorname{lgG}$ ) used to immunoprecipitate were: I = anti-HER-2 MAb-positive control; 2 =non-HIS, non-vaccinated BRG with growing tumour challenge; $3=$ non-vaccinated $r C D 34 ; 4=$ vaccinated rCD34; 5-7 = non-vaccinated, challenged rCD34; 8- $10=$ vaccinated and challenged rCD34. (C). In vitro effects of sera containing anti-HER-2 antibodies against HER-2-positive human cancer cells: growth inhibition (left panel) and antibody-dependent cellular cytotoxicity (ADCC, right panel). Mean and s.e.m. of five non-vaccinated (no vax) and six vaccinated (vax) rCD34 mice are shown $(P<0.05$ at Student's $t$-test $)$.

allogeneic recipients (Bornhauser et al, 2005). However, its lymphopoietic potential has never been directly compared with that of CD34 + HSC in a same experimental HIS model. In this study, the two types of cord blood human HSC-engrafted BALB/c $\mathrm{Rag2}^{-1-}$; Il2 $\mathrm{rg}^{-1-}$ mice showed similar levels and differentiation ability, giving about $50 \%$ human-circulating leukocytes at 5 weeks, long-term persistence of human cells in lymphoid organs and production of human antibodies. Noteworthy, in some regards, rCD133 mice showed a more robust human reconstitution although at early time points. Therefore, CD133 + cells show a partially different in vivo differentiation potential as compared with $\mathrm{CD} 34^{+}$cells and may provide a valuable alternative to $\mathrm{CD}^{+}{ }^{+}$cell population for xenotransplantation.

The HIS rCD34 and rCD133 mice were randomised to a nonvaccinated group and a group vaccinated with an adjuvanted cell vaccine, consisting of human HER-2-positive cancer cells combined with systemic IL-12 administration. At the end of two monthly vaccination cycles mice were able to produce specific anti-HER-2 IgG human antibodies, whereas did not show changes in any other parameter studied (such as percentage and differentiation of circulating human populations and total human antibodies)

Potency of vaccine is known to determine the intensity of the immune response, with adjuvants having important roles. Allogeneicity and recombinant IL-12 were previously needed as adjuvants in HER-2 cell vaccines to break tolerance to HER-2 in

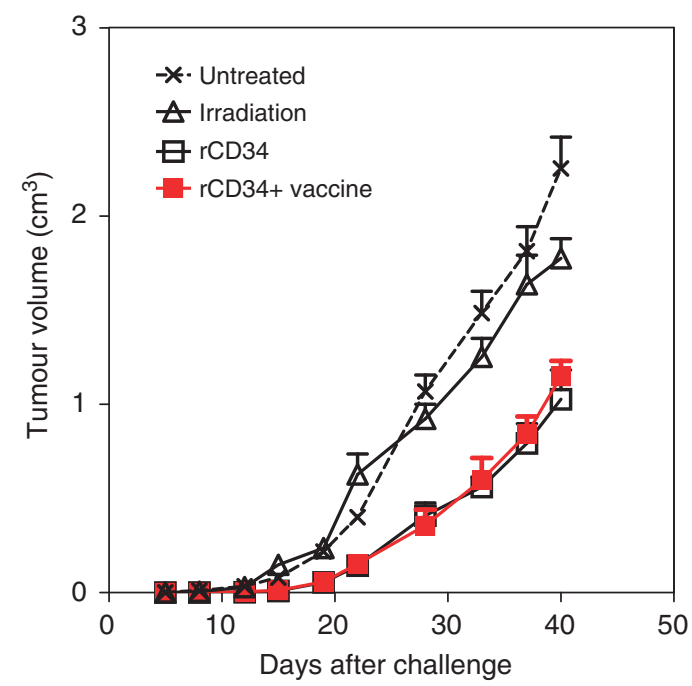

Figure 4 Inhibition of tumour growth of human HER-2-positive cancer cells in rCD34 mice vs control BRG mice (untreated or subjected to neonatal irradiation only). Mean tumour volumes and s.e. are shown ( $n=5-7$ for treated mice, $n=23$ for untreated mice). Tumour volumes observed in rCD34 mice (vaccinated or not) were statistically smaller than those of control groups (untreated or irradiated) starting from day $19(P<0.05$ at least at Student's t-test $)$

Table I Inhibition of lung metastases of human HER-2-positive cancer cells in vaccinated rCD34 mice

\begin{tabular}{lccccc}
\hline & & \multicolumn{4}{c}{ Lung metastases } \\
\cline { 3 - 6 } Mice treatment & Vaccination & Incidence & $\%$ & Median & Range \\
\hline Untreated & No & $10 / 12$ & 83 & 15 & $0-218$ \\
Irradiation & No & $5 / 6$ & 83 & 12 & $0-61$ \\
rCD34 & No & $6 / 6$ & 100 & 11 & $2-50$ \\
rCD34 & Yes & $3 / 7^{\text {a }}$ & 43 & $0^{\text {b }}$ & $0-20$ \\
\hline
\end{tabular}

${ }^{\mathrm{a}} \mathrm{p}=0.049$ at $\chi^{2}$ Fisher exact test $v$ s non-vaccinated $\mathrm{rCD} 34 .{ }^{\mathrm{b}} p=0.028$ at nonparametric Wilcoxon rank sum test vs untreated.

transgenic HER-2 mice, eliciting a specific anti-HER-2 humoral response (Nanni et al, 2001). In the present study, combination of IL-12 with a cell vaccine carrying the adjuvant allogeneicity, since matching between engrafting human HSC and HER-2-positive cancer cell vaccine is highly unlikely, was able to elicit a specific humoral response. This suggest that the unsatisfactory immune responses so far obtained in most HIS models, also due to an incomplete immune matching of murine and human cells and cytokines (Manz, 2007), can be at least partly overcome by the use of more powerful vaccines. The highest immune responses so far obtained with HIS mice were elicited by viral infections or viral vaccines (Gorantla et al, 2007; Kuruvilla et al, 2007; Strowig et al, 2009; Kwant-Mitchell et al, 2009a; Becker et al, 2010).

Then mice were challenged through the s.c. injection of the same live HER-2-positive cancer cells previously used as vaccine, to further evaluate the ability to obtain an immune response strong enough to lead to tumour rejection. Concerning the timing of vaccination, we chose to start the vaccinations as early as possible to vaccinate mice with the maximal engrafting levels and with the aim to perform two cycles of vaccination (corresponding to two months). Challenge was performed just after vaccination completion, when engraftment levels were still good. Tumour growth was actually delayed but inhibition was independent of vaccination and did not achieve tumour rejection. This could be due to escape mechanisms by tumour cells (Cavallo et al, 2011) as well as to an incomplete response by HIS mice. These data are in agreement 

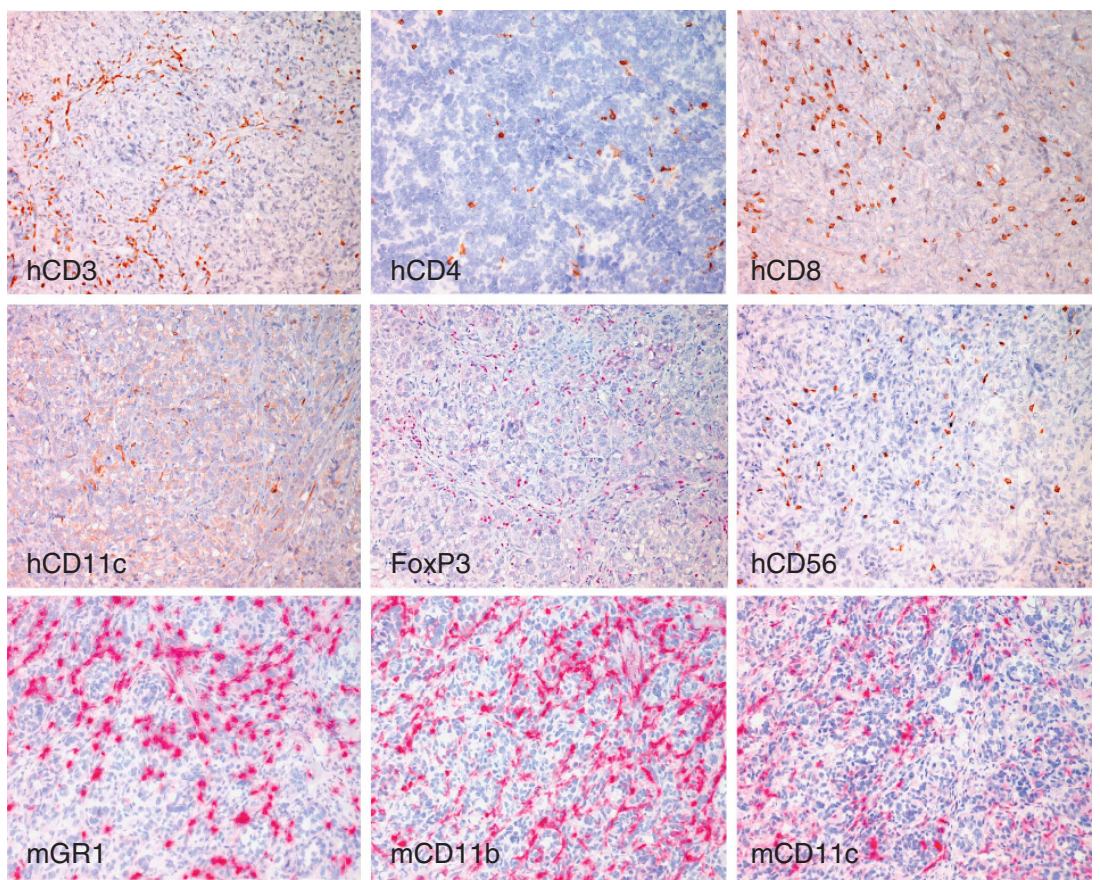

Figure 5 Human and murine tumour-infiltrating inflammatory cells. First two lines: immunohistochemistry with markers of human inflammatory cells: common marker of human T cells (hCD3 + in brown), helper T cells (CD4 + in brown), cytotoxic T cells (hCD8 + in brown), dendritic cells (hCDI Ic + in brown), regulatory T cells (hFoxp3 + in red) and NK cells (hCD56 + in brown). Third line: immunohistochemistry with markers of murine inflammatory cells: neutrophils (mGRI + in red), macrophages ( $m C D I I b+$ in red) and dendritic cells (mCDIIc + in red).

Table 2 Infiltrating human leucocytes in human tumours grown in rCD34 mice vaccinated or not

rCD34 individual mice

\begin{tabular}{|c|c|c|c|c|c|c|c|c|c|c|c|c|}
\hline $\begin{array}{l}\text { Tumour-infiltrating } \\
\text { human cells }\end{array}$ & & & & lo vaccin & & & & & Vaccine & & & 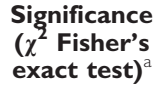 \\
\hline hCDIIC & + & + & - & + & + & - & +++ & + & - & ++ & ++ & $P=0.06$ \\
\hline hCD8 & +++ & +++ & - & ++ & +++ & +++ & $++1+++$ & ++ & $++/+++$ & +++ & ++ & NS \\
\hline hCD3 & ++ & +++ & - & +++ & +++ & +++ & +++ & +++ & +++ & +++ & ++ & NS \\
\hline hCDIIb & - & - & - & - & - & - & - & - & - & - & - & NS \\
\hline hCD56 & NP & ++ & NP & + & $\begin{array}{l}+1 \\
++\end{array}$ & $N P$ & ++ & ++ & $N P$ & NP & + & NS \\
\hline hCD4 & NP & ++ & NP & +++ & ++ & NP & ++ & ++ & NP & NP & ++ & NS \\
\hline Foxp3 & NP & NP & NP & ++ & +++ & NP & NP & +++ & NP & NP & +++ & NS \\
\hline
\end{tabular}

Abbreviations: $N P=$ not performed; NS = not significant. ${ }^{a}$ Low $(-/+)$ vs high $(++/+++)$ frequency of human cells.

with the finding that MHC-mismatched tumour cells can coexist with human HSC without rejection (Wege et al, 2011). However, the response elicited in vaccinated mice was able to almost completely abolish metastases.

Concerning antitumour immune activities of HIS mice, NK and cytotoxic T responses and production of IFN- $\gamma$ have been reported for a few non-viral tumour models (Kwant-Mitchell et al, 2009b; Wege et al, 2011), generally leading to an inhibition of tumour growth. However, very few data on immune responses elicited by antitumour vaccines have been reported (Kametani et al, 2006). Here, we have shown that in HIS mice an adjuvanted tumour cell vaccine can elicit specific antibody responses, along with an increased dendritic tumour cell infiltrate. These components, combined to the presence of NK cells and IFN- $\gamma$, could have a role in inhibiting metastasis in HIS mice. We cannot rule out that a human anti-MHC response (elicited by the mismatching between immune engrafted cells and vaccine) could also contribute to the inhibition of lung metastases. The immune response elicited was not able to neutralise a bulk injection of tumour cells, but was able to effectively control the day-by-day seeding of lung metastatic cells. Such data confirm a huge amount of experimental studies in murine systems showing that even partial immune responses can efficiently fight metastatic dissemination but hardly control growing tumours (Nanni et al, 2007). Migration and motility, which are known to have a major role in metastatic process, were inhibited by trastuzumab (Talukder et al, 2002; Yokotsuka et al, 2011). Similar effects could contribute to the dissociation of effects against tumour growth and metastases observed in our system.

This model could be exploited to obtain human monoclonal antibodies (Kametani et al, 2006; Becker et al, 2010) against tumour targets. In particular, our adjuvanted vaccine could lead to the development of new fully human anti-HER-2 monoclonal antibodies to integrate the therapeutic activity of those already entered into the clinic (Ross et al, 2009). A second interesting approach could be the study of the immunogenicity of different HER-2-positive cancer cell lines, in relation with different MHC haplotypes or with their natural as well as engineered cytokine production. SK-OV-3 cells produce several human cytokines, such as VEGF, IL-6, IL-8 and IL-15 (Melani et al, 1995; Keyes et al, 2003; Wang et al, 2010 and our unpublished data) that could interact 
A

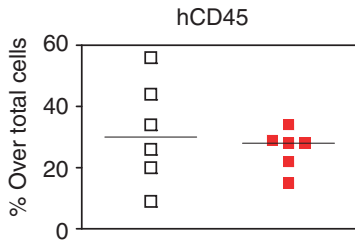

B

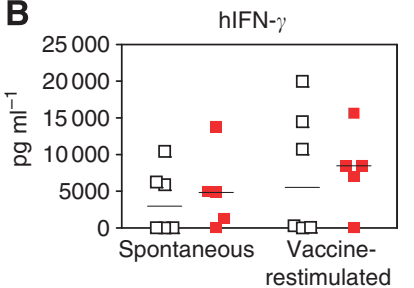

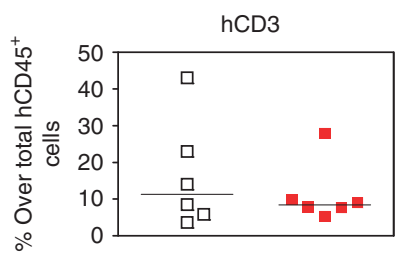
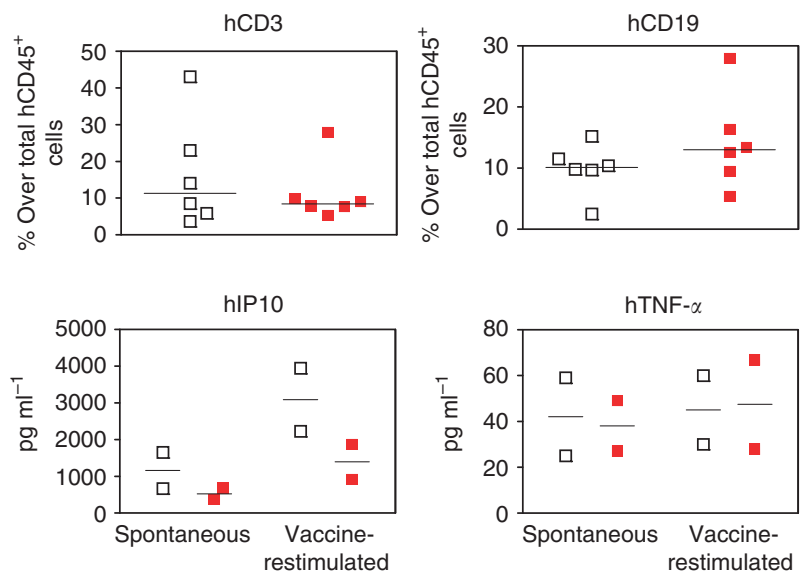

Figure 6 Human splenic engrafting and production of human cytokines by cells recovered from the spleen of HIS vaccinated or non-vaccinated mice after the challenge with live tumour cells. Total spleen cell yields $\left(\times 10^{6}\right)$ were: non-vaccinated, 6.2 \pm 1.3; vaccinated, $9.2 \pm$ I.8 (not significant at Student's t-test or non-parametric test). (A) Cytofluorometric evaluation of human differentiated populations. Open symbols: non-vaccinated; closed red symbols: vaccinated. Values of individual mice are shown. Continuous horizontal lines indicate median values. (B) Cytokine production by the spleen cells cultured in vitro for 6 days alone (spontaneous) or in the presence of proliferation-blocked vaccine cells (restimulated). Symbols as in $\mathbf{A}$.

with the human effectors engrafting HIS mice. The incomplete function of immune system in HIS mice has also been attributed to a lack of human-mouse cross-reactivity of several cytokines, as an example murine IL-6 is not active on human cells (Manz and Di Santo, 2009). IL-6 has pleiotropic activity ranging from immune suppression to induction of B-cell maturation (Dienz and Rincon, 2009). The production of some human cytokines by proliferationblocked (but live) SK-OV-3 vaccine cells, as well as by live SK-OV-3 challenge cells, could have had a role in the induction of the adaptive humoral response or in the immune responses observed in challenged HIS mice.

In conclusion, HIS mice were used to study the possibility of triggering in vivo a specific immune response against tumour antigens by human lymphocytes. Even though the immune response is still not complete, future refinements of this model could lead to a more realistic assessment of both the ability of a vaccine to induce immune responses in human lymphocytes and the efficacy (and limits) of the immune reactions elicited.

\section{ACKNOWLEDGEMENTS}

This work was supported by the Italian Association for Cancer Research (AIRC), Milan, Italy (project no. 10353); the Department of Experimental Pathology, University of Bologna (Pallotti funds); and the Italian Ministry for University and Research (PRIN 2008 project). Fellowships: Italian Foundation for Cancer Research, Milan, Italy (to AA); the University of Bologna (to SC and VS); and the Interdepartmental Center for Cancer Research 'Giorgio Prodi' ('W Vanini-S Cavagnino' fellowships to AP and AM).

Supplementary Information accompanies the paper on British Journal of Cancer website (http://www.nature.com/bjc)

\section{REFERENCES}

Becker PD, Legrand N, van Geelen CM, Noerder M, Huntington ND, Lim A, Yasuda E, Diehl SA, Scheeren FA, Ott M, Weijer K, Wedemeyer $\mathrm{H}$, Di Santo JP, Beaumont T, Guzman CA, Spits H (2010) Generation of human antigen-specific monoclonal IgM antibodies using vaccinated 'human immune system' mice. PLoS One 5(10): e13137

Bornhauser M, Eger L, Oelschlaegel U, Auffermann-Gretzinger S, Kiani A, Schetelig J, Illmer T, Schaich M, Corbeil D, Thiede C, Ehninger G (2005) Rapid reconstitution of dendritic cells after allogeneic transplantation of $\mathrm{CD133}$ + selected hematopoietic stem cells. Leukemia 19(1): 161-165

Brehm MA, Shultz LD, Greiner DL (2010) Humanized mouse models to study human diseases. Curr Opin Endocrinol Diabetes Obes 17(2): 120-125

Cavallo F, De Giovanni C, Nanni P, Forni G, Lollini PL (2011) 2011: the immune hallmarks of cancer. Cancer Immunol Immunother 60(3): $319-326$

De Giovanni C, Nicoletti G, Palladini A, Croci S, Landuzzi L, Antognoli A, Murgo A, Astolfi A, Ferrini S, Fabbi M, Orengo AM, Amici A, Penichet ML, Aurisicchio L, Iezzi M, Musiani P, Nanni P, Lollini PL (2009) A multi-DNA preventive vaccine for p53/Neu-driven cancer syndrome. Hum Gene Ther 20(5): 453-464

Dienz O, Rincon M (2009) The effects of IL-6 on CD4 T cell responses. Clin Immunol 130(1): 27-33

Drake AC, Khoury M, Leskov I, Iliopoulou BP, Fragoso M, Lodish H, Chen J (2011) Human CD34 CD133 hematopoietic stem cells cultured with growth factors including Angptl5 efficiently engraft adult NODSCID Il2rgamma (NSG) mice. PLoS One 6(4): e18382
Gorantla S, Sneller H, Walters L, Sharp JG, Pirruccello SJ, West JT, Wood C, Dewhurst S, Gendelman HE, Poluektova L (2007) Human immunodeficiency virus type 1 pathobiology studied in humanized BALB/ c-Rag2 - / - gammac - / - mice. J Virol 81(6): 2700-2712

Huntington ND, Di Santo JP (2008) Humanized immune system (HIS) mice as a tool to study human NK cell development. Curr Top Microbiol Immunol 324: 109-124

Kametani Y, Shiina M, Katano I, Ito R, Ando K, Toyama K, Tsukamoto H, Matsumura T, Saito Y, Ishikawa D, Taki T, Ito M, Imai K, Tokuda Y, Kato S, Tamaoki N, Habu S (2006) Development of human-human hybridoma from anti-Her-2 peptide-producing B cells in immunized NOG mouse. Exp Hematol 34(9): 1240-1248

Keyes KA, Mann L, Teicher B, Alvarez E (2003) Site-dependent angiogenic cytokine production in human tumor xenografts. Cytokine 21(2): 98-104

Koo GC, Hasan A, O’Reilly RJ (2009) Use of humanized severe combined immunodeficient mice for human vaccine development. Expert Rev Vaccines 8(1): 113-120

Kuruvilla JG, Troyer RM, Devi S, Akkina R (2007) Dengue virus infection and immune response in humanized $\operatorname{RAG} 2(-/-) \operatorname{gamma}(\mathrm{c})(-/-)$ (RAG-hu) mice. Virology 369(1): 143-152

Kwant-Mitchell A, Ashkar AA, Rosenthal KL (2009a) Mucosal innate and adaptive immune responses against herpes simplex virus type 2 in a humanized mouse model. J Virol 83(20): 10664-10676

Kwant-Mitchell A, Pek EA, Rosenthal KL, Ashkar AA (2009b) Development of functional human NK cells in an immunodeficient mouse model with the ability to provide protection against tumor challenge. PLoS One 4(12): e8379 
Legrand N, Ploss A, Balling R, Becker PD, Borsotti C, Brezillon N, Debarry J, de Jong Y, Deng H, Di Santo JP, Eisenbarth S, Eynon E, Flavell RA, Guzman CA, Huntington ND, Kremsdorf D, Manns MP, Manz MG, Mention JJ, Ott M, Rathinam C, Rice CM, Rongvaux A, Stevens S, Spits H, Strick-Marchand $\mathrm{H}$, Takizawa $\mathrm{H}$, van Lent AU, Wang $\mathrm{C}$, Weijer $\mathrm{K}$, Willinger $\mathrm{T}$, Ziegler $\mathrm{P}$ (2009) Humanized mice for modeling human infectious disease: challenges, progress, and outlook. Cell Host Microbe 6(1): 5-9

Lollini PL, Nicoletti G, Landuzzi L, Cavallo F, Forni G, De Giovanni C, Nanni P (2011) Vaccines and other immunological approaches for cancer immunoprevention. Curr Drug Targets 12(13): 1957-1973

Manz MG (2007) Human-hemato-lymphoid-system mice: opportunities and challenges. Immunity 26(5): 537-541

Manz MG, Di Santo JP (2009) Renaissance for mouse models of human hematopoiesis and immunobiology. Nat Immunol 10(10): 1039-1042

Meek B, Cloosen S, Borsotti C, Van Elssen CH, Vanderlocht J, Schnijderberg MC, van der Poel MW, Leewis B, Hesselink R, Manz MG, Katsura Y, Kawamoto H, Germeraad WT, Bos GM (2010) In vitrodifferentiated $\mathrm{T} /$ natural killer-cell progenitors derived from human CD34 + cells mature in the thymus. Blood 115(2): 261-264

Melani C, Pupa SM, Stoppacciaro A, Menard S, Colnaghi MI, Parmiani G, Colombo MP (1995) An in vivo model to compare human leukocyte infiltration in carcinoma xenografts producing different chemokines. Int J Cancer 62(5): 572-578

Nanni P, Nicoletti G, De Giovanni C, Landuzzi L, Di Carlo E, Cavallo F, Pupa SM, Rossi I, Colombo MP, Ricci C, Astolfi A, Musiani P, Forni G, Lollini PL (2001) Combined allogeneic tumor cell vaccination and systemic interleukin 12 prevents mammary carcinogenesis in HER-2/neu transgenic mice. J Exp Med 194(9): 1195-1205

Nanni P, Nicoletti G, Landuzzi L, Croci S, Murgo A, Palladini A, Antognoli A, Ianzano ML, Stivani V, Grosso V, Maira SM, Garcia-Echeverria C, Scotlandi K, De Giovanni C, Lollini PL (2010) High metastatic efficiency of human sarcoma cells in Rag2/gammac double knockout mice provides a powerful test system for antimetastatic targeted therapy. Eur J Cancer 46(3): 659-668

Nanni P, Nicoletti G, Palladini A, Croci S, Murgo A, Antognoli A, Landuzzi L, Fabbi M, Ferrini S, Musiani P, Iezzi M, De Giovanni C, Lollini PL (2007) Antimetastatic activity of a preventive cancer vaccine. Cancer Res 67(22): 11037-11044

Nomura T, Tamaoki N, Takakura A, Suemizu H (2008) Basic concept of development and practical application of animal models for human diseases. Curr Top Microbiol Immunol 324: 1-24

Ross JS, Slodkowska EA, Symmans WF, Pusztai L, Ravdin PM, Hortobagyi GN (2009) The HER-2 receptor and breast cancer: ten years of targeted anti-HER-2 therapy and personalized medicine. Oncologist 14(4): 320-368

Rossi L, Manfredini R, Bertolini F, Ferrari D, Fogli M, Zini R, Salati S, Salvestrini V, Gulinelli S, Adinolfi E, Ferrari S, Di Virgilio F, Baccarani M, Lemoli RM (2007) The extracellular nucleotide UTP is a potent inducer of hematopoietic stem cell migration. Blood 109(2): 533-542

Shultz LD, Ishikawa F, Greiner DL (2007) Humanized mice in translational biomedical research. Nat Rev Immunol 7(2): 118-130

Strowig T, Gurer C, Ploss A, Liu YF, Arrey F, Sashihara J, Koo G, Rice CM, Young JW, Chadburn A, Cohen JI, Munz C (2009) Priming of protective $\mathrm{T}$ cell responses against virus-induced tumors in mice with human immune system components. J Exp Med 206(6): 1423-1434

Talukder AH, Bagheri-Yarmand R, Williams RR, Ragoussis J, Kumar R, Raz A (2002) Antihuman epidermal growth factor receptor 2 antibody herceptin inhibits autocrine motility factor (AMF) expression and potentiates antitumor effects of AMF inhibitors. Clin Cancer Res 8(10): 3285-3289

Thiel U, Pirson S, Muller-Spahn C, Conrad H, Busch DH, Bernhard H, Burdach S, Richter GH (2011) Specific recognition and inhibition of Ewing tumour growth by antigen-specific allo-restricted cytotoxic T cells. Br J Cancer 104(6): 948-956

Traggiai E, Chicha L, Mazzucchelli L, Bronz L, Piffaretti JC, Lanzavecchia A, Manz MG (2004) Development of a human adaptive immune system in cord blood cell-transplanted mice. Science 304(5667): 104-107

Wang Y, Niu XL, Qu Y, Wu J, Zhu YQ, Sun WJ, Li LZ (2010) Autocrine production of interleukin-6 confers cisplatin and paclitaxel resistance in ovarian cancer cells. Cancer Lett 295(1): 110-123

Wege AK, Ernst W, Eckl J, Frankenberger B, Vollmann-Zwerenz A, Mannel DN, Ortmann O, Kroemer A, Brockhoff G (2011) Humanized tumor mice - a new model to study and manipulate the immune response in advanced cancer therapy. Int J Cancer 129(9): 2194-2206

Wei WZ, Jacob J, Radkevich-Brown O, Whittington P, Kong YC (2008) The 'A, B and C' of Her-2 DNA vaccine development. Cancer Immunol Immunother 57(11): 1711-1717

Yokotsuka M, Iwaya K, Saito T, Pandiella A, Tsuboi R, Kohno N, Matsubara O, Mukai K (2011) Overexpression of HER2 signaling to WAVE2-Arp2/3 complex activates MMP-independent migration in breast cancer. Breast Cancer Res Treat 126(2): 311-318

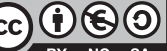

This work is licensed under the Creative Commons Attribution-NonCommercial-Share Alike 3.0 Unported License. To view a copy of this license, visit http://creativecommons.org/ licenses/by-nc-sa/3.0/ 